\title{
A New Coronavirus-Like Particle Associated With Diarrhea in Swine ${ }^{1}$
}

\section{Brief Report}

\author{
By \\ M. B. Pensaert and P. de Bouck \\ Laboratory of Virology, Faculty of Veterinary Medicine University of Gent, \\ Gent, Belgium
}

With 2 Figures

Accepted June 26, 1978

\section{Summary}

Coronavirus-like particles were detected by electron microscopy in the intestinal contents of pigs during a diarrheal outbreak on 4 swine breeding farms. Diarrhea was reproduced in experimental pigs with one of the isolates, designated CV 777, which was found to be distinct from the 2 known porcine coronaviruses, transmissible gastroenteritis virus and hemagglutinating encephalomyelitis virus.

In 1946, Dorle and Hotchnos (2) described a viral diarrhea in swine and called it transmissible gastroenteritis. Until recently, transmissible gastroenteritis virus was the only virus known to be specifically associated with diarrhea in swine of all ages. In 1976, following the discovery of rotaviruses in different animal species, a porcine rotavirus was detected in the feces of pigs with diarrhea (14). Diarrhea could be reproduced experimentally in piglets with this virus. In a search for rotaviruses on Belgian swine breeding farms with diarrheal problems, a. new coronavirus-like particle was detected by electron microscopic examination of intestinal or fecal samples from sick pigs. The present report deseribes the morphology of this coronavirus-like particle, and shows that it is distinct from the known porcine coronaviruses and causes diarrhea.

Up to now, the only coronaviruses isolated from swine have been transmissible gastroenteritis virus (TGEV) and hemagglutinating encephalomyelitis virus (HEV). TGEV has been described as a cause of diarrhea in swine in countries all over the world (13). Numerous studies have been performed on the virus-animal interactions of TGEV, which is usually detected either by its isolation from fecal

I These studies were supported by the Institute for the Encouragement of Research in Industry and Agriculture (TWONL), Brussels, Belgium. 
material in cell cultures or by immunofluorescence in the small intestinal epithelium of infected pigs $(7,12)$. TGEV infections can also be diagnosed serologically. HEV was first described in Canada in 1962 as a cause of central nervous disorders in pigs (4). The same virus was later associated with a disease syndrome called vomiting and wasting disease in several European countries $(1,8)$. The virus can easily be detected by cultivation in several porcine cell cultures (11). Both TGEV and HEV have been classified as coronaviruses mainly on the basis of their specific morphology (10).

In 1977, a sudden outbreak of diarrhea was observed in swine of all ages on 4 Belgian swine breeding farms. The morbidity in sows was very variable and the animals recovered after a diarrhea which lasted 3 to 4 days. All the pigs showed a watery diarrhea. Death occurred up to the age of 7 days and the overall mortality rate in these piglets was approximately 50 per cent (9). It decreased with increasing age. TGEV was suspected as the cause of this diarrhea. However, the direct immunofluorescence test for the diagnosis of TGEV, which is routinely applied on cryostat sections of the small intestine of sick pigs, was negative for these pigs. The absence of seroneutralizing antibodies to TGEV in the blood of sows collected 6 to 12 weeks after the outbreak confirmed that TGEV was not involved.

In an attempt to arrive at an etiologic diagnosis, fecal material and intestinal contents from pigs of each farm were subsequently processed for examination in an electron microscope by negative staining. They were diluted 1 to $5(\mathrm{v} / \mathrm{v})$ in phosphate-buffered saline, pH 7.3 and clarified at $3000 \times g$, at $4^{\circ} \mathrm{C}$, for 30 minutes. The supernatant was layered on top of a 20 per cent sucrose solution and centrifuged at $150,000 \times g$, at $4^{\circ} \mathrm{C}$, for 40 minutes. The resulting pellet was resuspended in a few drops of distilled water, placed on 200 mesh formvar coated grids, and stained with 2 per cent $\mathrm{K}$-phosphotungstate, $\mathrm{pH}$ 6.1. Grids were examined using a Zeiss EM 9 S-2 electron microscope at an acceleration voltage of $60 \mathrm{KV}$. Micrographs used for particle size measurement were taken at an instrumental magnification of $28,000 \times$, which were then photographically enlarged to $84,000 \times$ or $168,000 \times$.

Rotavirus particles were not detected. However, coronavirus-like particles were observed in specimens of pigs from each of the 4 breeding farms. One of the fecal samples containing these coronavirus-like particles was designated CV 777 and was used for further studies.

The etiologic relationship between the coronavirus-like partieles, CV777, and the occurrence of diarrhea was established by oral inoculation of a 20 per cent suspension of the fecal material contaming CV777 into a one day old colostrumdeprived pig. The experimental pig was killed 30 hours later, at the height of diarrhea, and a virus stock was prepared from an homogenate of its small intestine and contents. A bacteria free filtrate of the supernatant of a 20 per cent suspension of this material was used for inoculation of 12 colostrum-deprived-hysterectomy-derived piglets, kept in isolation. Seven control pigs were used. The pigs were inoculated at the age of 3 to 15 days. All the inoculated pigs developed a watery diarrhea within 24 to 36 hours after inoculation whereas the control animals remained normal. Coronavirus-like particles were detected by electron microscopic examination in the watery feces or intestinal contents of each of the 
experimentally inoculated pigs. Such particles were not found in the feces of the same pigs prior to inoculation or in the fecal samples of the control animals.

The particles, shown in Figure 1, had typical coronavirus morphology. They were pleomorphic with a range in diameter of 95 to $190 \mathrm{~nm}$, including the projections, which were approximately $18 \mathrm{~nm}$ in length. Most particles were between 130 and $170 \mathrm{~nm}$ in diameter. The projections formed a single fringe radiating from the core. They appeared to be club-shaped. Only the dilated distal ends of the projections were seen on the micrographs. The negative stain also appeared to settle on the surface of some particles and an electron opaque central area covered by surface projections was often seen (Fig. 1a-arrows and 1c). No internal structure was observed. It was impossible to distinguish these coronavirus-like particles morphologically from TGEV or HEV particles from similar preparations.
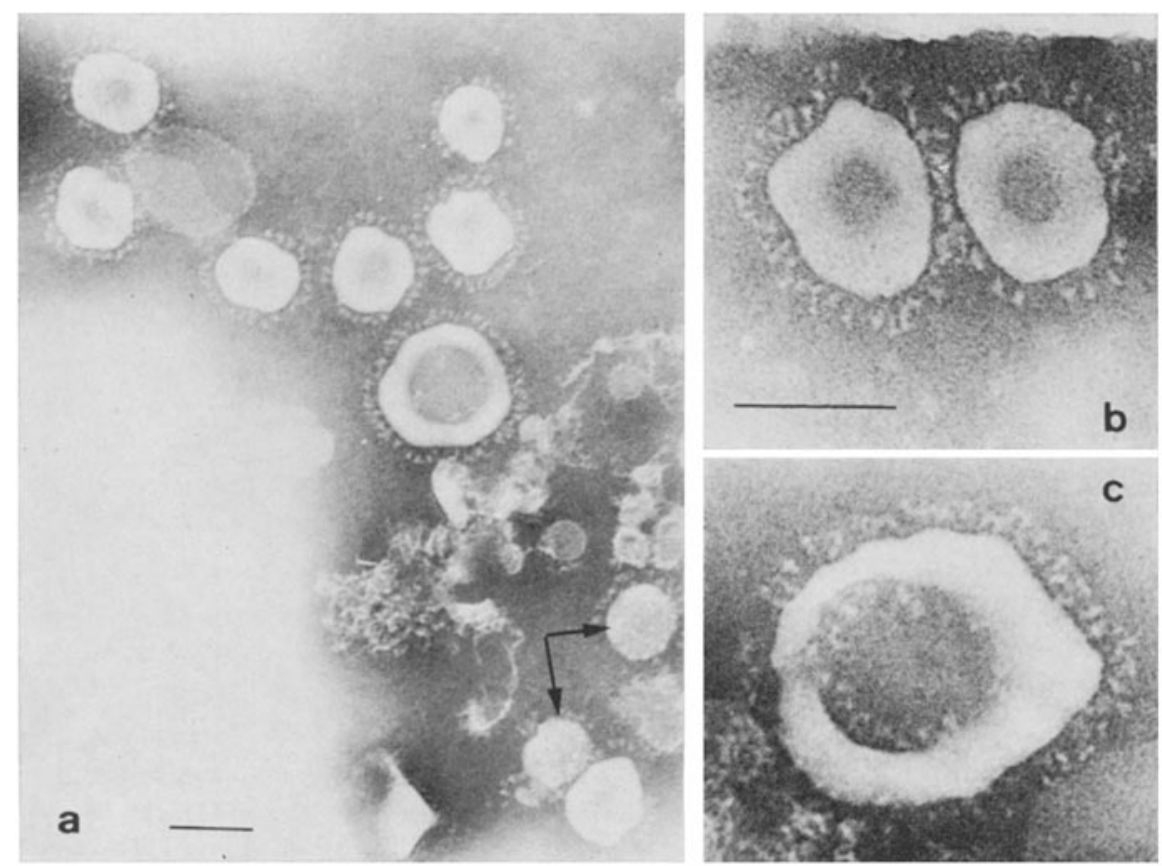

Fig. 1. Negatively stained coronavirus-like particles CV 777. Bar represents $100 \mathrm{~nm}$

Other particles, different from these coronavirus-like particles, were also observed in the majority of the fecal samples. As seen in Figure 2, they were pleomorphic and very variable in size, ranging in diameter from 95 to $650 \mathrm{~nm}$ with an average diameter of 190 to $225 \mathrm{~nm}$. They carried numerous short projections, of approximate length $9 \mathrm{~nm}$, on their surfaces. Similar particles of unknown identity have been described in human and animal fecal samples $(3,5,6)$. In the present studies such particles have also been found in the solid fecal samples of the control pigs. They appeared, therefore, not to be associated with diarrhea. Rotaviruses and other recognizable virus particles were not seen in control or experimentally inoculated pigs. 
As already mentioned, TGEV was eliminated as the cause of the diarchea on the original farms. Additionally 9 out of the 12 experimentally inoculated pigs, killed at the heigth of diarrhea, were negative for TGE viral antigens in their small intestinal epithelium by the direct immunofluorescence test. Furthermore, the remaining 3 pigs, inoculated with $\mathrm{CV} 777$ at the age of 15 days, were allowed to recover after a diarrhea which lasted $4-5$ days. A serum sample, collected from these pigs 3 weeks later, did not contain neutralizing antibodies against the cell culture adapted Purdue strain of TGEV.

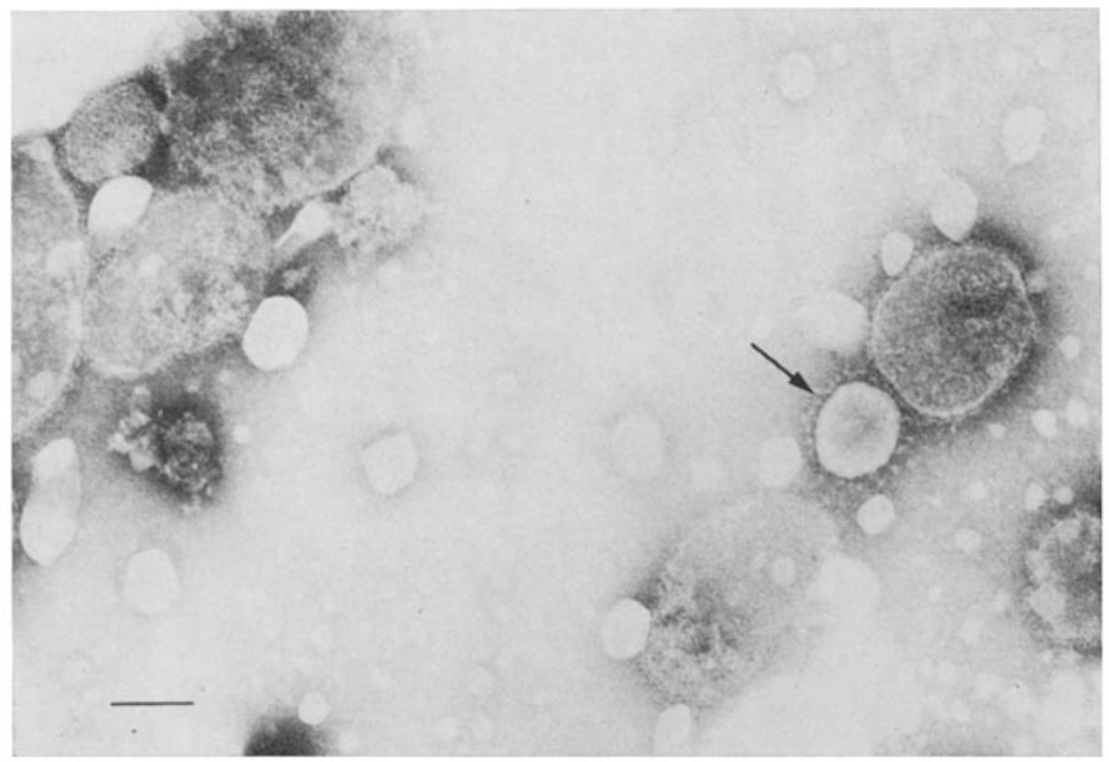

Fig. 2. One coronavirus-like particle CV 777 (arrow) together with pleomorphic particles of unknown identity. Bar represents $100 \mathrm{~nm}$

The possibility that the CV777 particles consisted of HEV was less likely since the latter virus does not cause diarrhea in pigs. Cryostat sections of the small intestine of experimentally inoculated pigs were negative for fluorescence by the direct test using a conjugate directed against the VW572 isolate of HEV (8). Furthermore, the pigs that had been allowed to recover did not possess hemagglutination-inhibiting or seroneutralizing antibodies against this $\mathrm{HEV}$ isolate.

Preliminary attempts were made to cultivate the coronavirus-like particle, CV 777, in primary pig kidney cell cultures and in secondary porcine thyroid cells. Four weekly blind passages were made. The cells were examined for cytopathic effect and hemadsorption with chicken red blood cells, and the cell culture fluids were examined for hemagglutination. No evidence of viral replication in the cell cultures was obtained. It is known that the HEV can easily be isolated in primary pig kidney cell cultures using the same criteria (8).

The present data suggest that, as well as TGEV and HEV, another previously unrecognized coronavirus-like virus is prevalent in swine. The results indicate that diarrhea can be reproduced in experimental pigs with this virus and that it is 
associated with certain outbreaks of epizootic diarrhea on Belgian swine breeding farms.

More details on the clinical disease in the field and on the results of the experimental infections will be reported later.

\section{Iheferences}

1. Cartwright, S. F., Lucas, M., Cavill, J. P., Gush, A. F., Blandford, T. B.: Vomiting and wasting disease of piglets. Vet. Rec. 84, 175-176 (1969).

2. Doyle, L. P., Hutohings, L. M.: A transmissible gastroenteritis in pigs. J. Amer. vet. Assoe. 108, 257-259 (1946).

3. Fuwwert, T. H., Boxalt, E. : The hunt for viruses in infections of the alimentary systern: an immuno electronmicroscopical approach. Clinics in Gastroenterology 5, $359-385(1976)$.

4. Greig, A. S., Mrtcheit, D., Corner, A. H., Bannister, G. L., Meads, E. B., JULIAN, R. J.: A hemagglutinating virus producing encephalomyelitis in baby pigs. Canad. J. comp. Med. vet. Sci. 26, $49-56$ (1962).

5. Mathan, M., Mathan, V. I., Swaminathan, S. P., Yksudoss, S., Baker, S. J.: Pleomorphic virus-like particles in human faeces. Lancet 1, 1068-1069 (1975).

6. Monulty, M. S., Currax, W. L., McFerrai, J. B.: Virus-like particles in calves' faeces. Lancet 2, 78-79 (1975).

7. Pensaert, M. B., Hafluerman, E. O., Burnstein, T. : Diagnosis of transmissible gastroenteritis in pigs by immunofluorescence. Canad. J. comp. Med. 32, 555-561 (1968).

8. Pensayrt, M. B., Callebaut, P. E.: Characteristies of a coronavirus causing vomition and wasting in pigs. Arch. ges. Virusforsch. 44, 35-50 (1974).

9. Pensaert, M., de Boucz, P., De Roose, P.: A virus isolated from an apparently new epizootic diarrhea in swine. Proe. 5th World Int. Pig Vet. Congress, Zagreb, Yugoslavia, June 1978, Abstract K. A. 9.

10. Philip, J. I. H., Cartwright, S. F., SCott, A. C.: The size and morphology of transmissible gastroenteritis and vomiting and wasting disease viruses of pigs. Vet. Rec. 88, 311-312 (1971).

11. Pirtle, E. C.: Titration of two porcine respiratory viruses in mammalian cell cultures by direct fluoreseent antibody staining. Amer. J. vet. Res. 35, 249-250 (1974).

12. WrTte, K. H.: Isolation of the virus of transmissible gastroenteritis (TGE) from naturally infected piglets in cell culture. Zbl. Vet. Med. B 18, 770 (1971).

13. Woode, G. N.: Transmissible gastroenteritis of swine. The Vet. Bull. 39, 239 (1969).

14. Woode, G. N., Bridger, J., HAIL, G. A., Jones, J. M., JACkson, G.: The isolation of reovirus-like agents (rotaviruses) from acute gastroenteritis of piglets. J. med. Microbiol. 9, 203-209 (1976).

Authors' address: Prof. Dr. M. Pensaent, Laboratory of Virology, Faculty of Veterinary Medicine, University of Gent, Casinoplein 24, B-9000 Gent, Belgium.

Received May 22, 1978 